\title{
Two Psychological Interventions Are Effective in Severely Disabled, Chronic Back Pain Patients: A Randomised Controlled Trial
}

\author{
Julia Anna Glombiewski • Jens Hartwich-Tersek • \\ Winfried Rief
}

Published online: 2 December 2009

(C) International Society of Behavioral Medicine 2009

\begin{abstract}
Background Many pain patients appreciate biofeedback interventions because of the integration of psychological and physiological aspects. Therefore we wanted to investigate in a sample of chronic back pain patients whether biofeedback ingredients lead to improved outcome of psychological interventions.

Method One hundred and twenty-eight chronic back pain patients were randomly assigned to cognitive-behavioural therapy (CBT), cognitive-behavioural therapy including biofeedback tools (CBT-B) or waitlist control (WLC). The sample was recruited from a highly disabled group including many patients with low education status and former back surgeries. Measures on pain, physical functioning, emotional functioning, coping strategies and health care utilisation were taken at pretreatment, posttreatment and 6 months of follow-up.

Results The results indicated significant improvements on most outcome measures for CBT-B and CBT in comparison to WLC. CBT-B and CBT were equally effective (e.g. ITT effect sizes for pain intensity: CBT-B, 0.66 (95\% CI 0.39-0.95); CBT, 0.60 (95\% CI 0.33-0.87)).

Conclusion In conclusion, biofeedback ingredients did not lead to improved outcome of a psychological intervention. Cognitive-behavioural treatment as a "package" of respondent, operant and cognitive interventions was effective for ameliorating pain-related symptoms for chronic back pain
\end{abstract}

The study was supported by a doctoral thesis scholarship from the University of Marburg.

J. A. Glombiewski $(\bowtie) \cdot J$. Hartwich-Tersek $\cdot$ W. Rief

Section for Clinical Psychology and Psychotherapy,

Philipps-University of Marburg,

Gutenbergstr. 18,

35032 Marburg, Germany

e-mail: julia.glombiewski@staff.uni-marburg.de patients treated in an outpatient setting. The high treatment acceptability associated with biofeedback ingredients can also be achieved with pure psychological interventions.

Keywords Chronic pain - Chronic low back pain .

Biofeedback · Cognitive-behavioural treatment .

Psychological intervention

\section{Introduction}

Chronic back pain affects $20 \%$ of adults in Germany [1]. Cognitive-behavioural treatment is known to be one of the most effective interventions for this condition [2], although the effect sizes are only moderate.

Most cognitive-behavioural treatment studies for chronic back pain used a 'package' approach including different treatment modalities. As van Tulder et al. [3] state, "little is known about the actual or comparative value of different methods within cognitive-behavioural treatment for chronic back pain" and "it is still unclear which type of behavioural treatment is most effective, which components are necessary, and which are superfluous." Our study was designed to address these important questions.

Biofeedback is a very popular intervention within pain treatments. As a stand-alone treatment or with other treatment modalities, it has been found to be beneficial in the rehabilitation of chronic back pain in some studies [4-9]. However, other studies question advantages of biofeedback-oriented treatments [10-13].

Only two studies have dealt with the direct comparison of biofeedback-based cognitive-behavioural therapy including biofeedback tools (CBT-B) and cognitive-behavioural treatment (CBT) in chronic back pain, arriving at contradictory conclusions. Flor and Birbaumer [5] demonstrated that 
electromyography (EMG) biofeedback was significantly more effective than cognitive-behavioural therapy or conservative medical treatment at 6-month and 2-year follow-ups. In contrast, Newton-John et al. [7] found no significant difference between CBT and EMG biofeedback. The authors concluded that their findings could be limited due to including only mildly disabled patients and a very small sample size. Thus, it still remains unclear how or even whether back pain patients profit from the inclusion of biofeedback within cognitive-behavioural treatments.

The present study was designed to examine effects of biofeedback as intervention within outpatient cognitivebehavioural treatment of chronic back pain. To our knowledge, it was the first randomised controlled clinical trial to address this specific question. Based on the results reported by Flor et al. [5], we hypothesised (hypothesis "B") that participants treated with CBT-B would report more treatment gains than patients treated with CBT concerning principal outcome measures of pain and treatment satisfaction at posttreatment and 6 months of follow-up.

To test the general effectiveness of treatment, we additionally hypothesised (hypothesis "A") that CBT and CBT-B for chronic back pain would be effective in comparison to waitlist control (WLC) [14].

\section{Method}

The study was conducted following CONSORT guidelines and guidelines for rating the quality of psychological trials for pain published by Yates et al. [15].

\section{Sample Size Estimation}

To detect effects of $d=0.2$ (repeated measures analysis of covariance, within-between interaction) with $95 \%$ power we estimated a total sample size of $N=86(n=43$ per group). We expected small effect size according to Cohen [16] because we compared two active treatments. The estimations were calculated with g*power [17]. Taking possible missing data and dropout into account, we aimed for a sample size of 120 .

\section{Participants}

Participants were 128 adults form Marburg and surroundings. Marburg is a German university city with a population of around 70,000. The patients were medical care seekers, referred over a 3-year period from two outpatient anaesthesiology centres $(54.3 \%)$ and several general practitioners' offices in Marburg (31.3\%) or self-referrals through media advertisements in local newspapers (14.4\%). The cooperating anaesthesiologists and general practitioners were informed about inclusion criteria (checklist) and notified their patients about the possibility to participate in the study. This sampling procedure reflects clinical practice in Germany, where general practitioners or outpatient medical specialists can refer patients to outpatient psychological treatment. Inclusion criteria were as follows: musculoskeletal pain of the low back, mid back or upper back as a major complaint, and pain duration of at least 6 months on most days of the week. Exclusion criteria were: severe alcohol or drug abuse, psychotic disorder, organic brain damage and ongoing psychological treatment. To ensure high clinical relevance through low selection bias, we did not define further exclusion criteria.

\section{Therapists and Treatment Fidelity}

The four therapists were one female licensed cognitivebehavioural therapist (treated five of the 116 intention-totreat patients $=4.3 \%$ ) and two female (one of them treated 44 patients $=37.9 \%$, the other 16 patients $=13.8 \%$ ) and one male (treated 51 patients $=44 \%$ ) Ph.D. students and advanced trainees in cognitive-behavioural therapy. Patients were randomly assigned to therapists. At the end of the study randomisation was interrupted by replacing the first author by another female therapist at the end of the study who treated 16 patients.

All therapists were trained in a standardised treatment plus biofeedback and were supervised weekly by licensed cognitive-behavioural therapists and supervisors. Treatments were described in detailed session-by-session protocols to maintain treatment fidelity. Selected videotapes of therapy sessions were discussed during supervision contacts to ensure adherence to protocols.

Procedure

The study was approved by the German Psychological Society Ethics Committee and registered in a public trials registry (trial code: NCT00454064) (http://www.clinicaltrials. gov). According to ethical requirements, all subjects were informed about the nature of the study and written informed consent was obtained. Patient recruitment began in September 2004. The last 6 months of follow-up measures were requested in January 2008.

The study was conducted at an outpatient clinic for psychological interventions at the University of Marburg, Germany. One hundred and forty patients contacted the clinic about the stud. A semi-structured interview, conducted by one of the therapists, which took about $1 \mathrm{~h}$ to complete, was carried out to determine eligibility for the study. Twelve patients were excluded due to not meeting inclusion criteria (Fig. 1). 


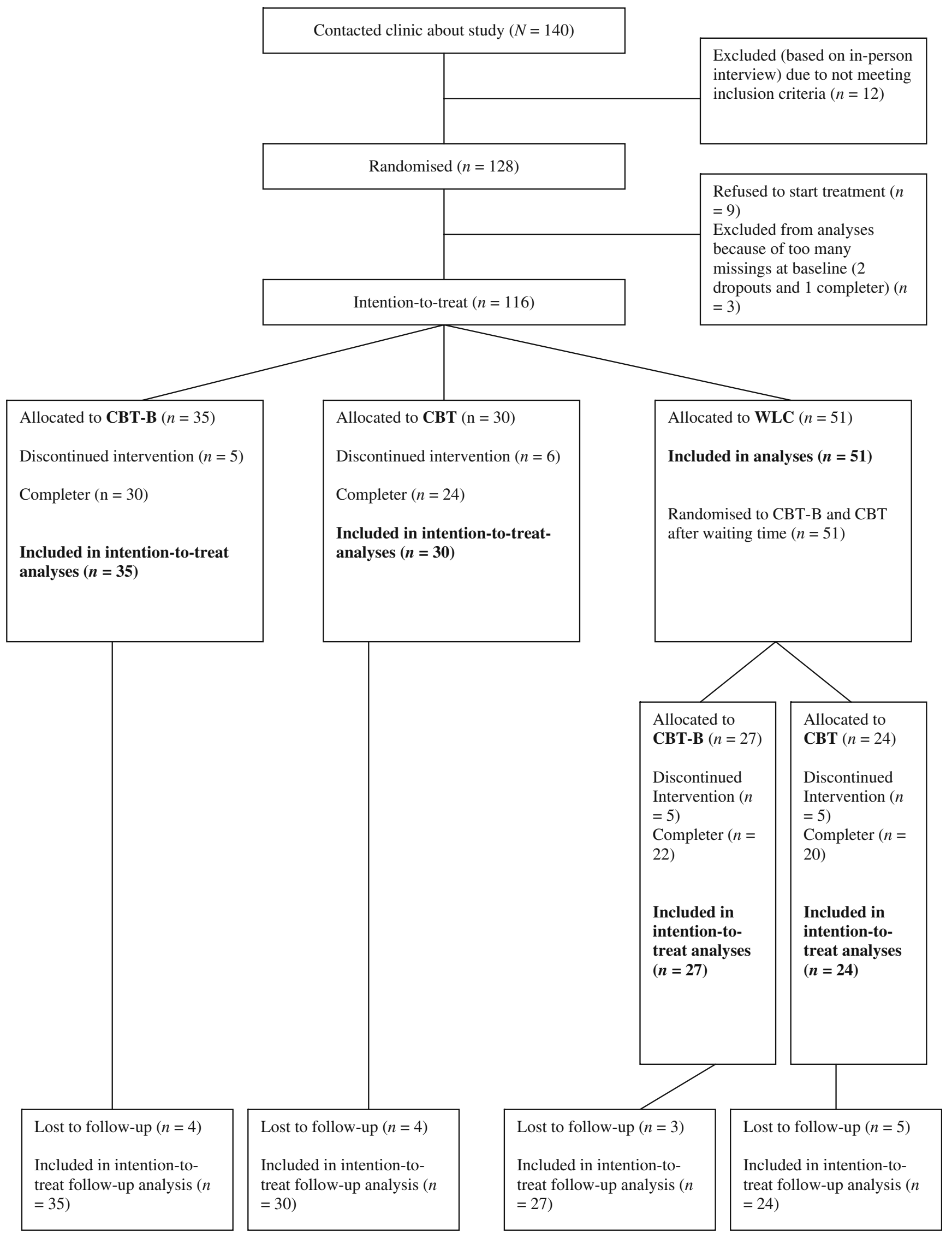

Fig. 1 Flow of the participants through the study 
After the patients had given their consent to participate in the study, they were randomly assigned to either WLC, CBT or CBT-B. The randomisation method was simple randomisation (random number generator) performed by a person who was not involved in assessment or treatment delivery. The allocation process was coordinated by the first author and occurred before the study information took place. There were two waves of randomization. In a first wave, participants were randomised over the three conditions (WLC, CBT-B and CBT). In a second wave, participants that were allocated to the WLC and who had waited 4 months were randomised over the two treatment conditions. Both active treatments consisted of 25 1-h sessions (including two 1-h diagnostic interviews conducted at the beginning of treatment), conducted weekly in an individualised format. Treatment was defined as complete when 17 sessions were attended. The average duration of treatment was 8 months. There were no differences between the two interventions groups on the average length of treatment. This length of psychological treatment is typical in Germany (so-called short-term psychotherapy) and was chosen to ensure the generalisability of outcomes by measuring the effects of treatment under "real world" clinical settings. Measures were taken at pretreatment (and additionally 4 months prior to treatment for the WLC group), at posttreatment and at 6 months of follow-up.

\section{Treatments}

Cognitive-behavioural therapy The initial phase of the programme included information about biopsychosocial aspects of pain (sessions 1 and 2), goal setting (session 3 ), progressive muscle relaxation (sessions 4, 5 and the first 15 min of most of the following sessions), activity scheduling (sessions 6-8 and 13), cognitive therapy for restructuring pain cognitions (sessions 9 and 10), restructuring of fear avoidance beliefs (session 12), breathing exercises and further progressive muscle relaxation training (sessions 11 and 15), attention diversion (session 14), relapse prevention strategies (session 17 and the last session) and stress-coping skills.

Cognitive-behavioural therapy and biofeedback The treatment was identical to CBT, but many of the interventions were supported by biofeedback ( $40 \%$ of treatment time), mainly EMG biofeedback. The aims of the biofeedback intervention were as follows: (a) to reduce tension in specific muscles, (b) to increase generalised relaxation and (c) to increase self-efficacy. Skin-conductance-level biofeedback and respiratory biofeedback were also occasionally offered for demonstration purposes, but the main goal for every patient was to achieve self-control by learning muscle self-control (through EMG biofeedback). The protocol of using biofeedback was completely standardised to ensure that the sessions will be comparable between subjects. For every session a protocol existed, where the therapist had to state if he or she exactly followed it. In sessions 1 and 2, the effect of stress on muscle tension and skin conductance level was demonstrated using visual feedback of bodily reactions. In sessions 4, 5, 11 and 15, patients practised muscle relaxation while visual EMG feedback and skin-conductance-level feedback was provided. The rationale of progressive muscle relaxation was also offered. In EMG feedback, up to four EMG electrodes were attached to painful areas of the back (over trapezius and erector spinae muscle groups). Patients exercised in sitting and standing positions and also while recalling stressful events; during these exercises, they tried to modify the physiological changes that accompanied the stress reaction [18]. Feedback trials were interspersed with no-feedback trials to facilitate the generalisation of the physiological relaxation response. Patients were encouraged to practise progressive muscle relaxation or any other relaxation technique to achieve lower levels of muscle tension. In sessions 11 and 15 , breathing techniques were introduced while respiratory feedback was given. In sessions 9 and 10 , the impact of cognitions on muscle tension and skin conductance level was demonstrated using EMG biofeedback and respiratory biofeedback. In session 12, dynamic EMG training [19] was applied (EMG biofeedback). Patients were also encouraged to exercise relaxation at home. CBT-B and CBT sessions were designed to have the same duration to have equal doses of therapy time and attention in both conditions.

The manual is under preparation for publication in German language ${ }^{1}$.

\section{Measures}

There were four assessment times: 4 months before starting treatment (only for subjects who were allocated to WLC), at pretreatment, at posttreatment and at 6-month follow-up (for all subjects). We chose outcome measures based on the recommendations of the Initiative on Methods, Measurement and Pain Assessment in Clinical Trials (IMMPACT) [14].

Pain measures Pain intensity was assessed with two measures. As the primary outcome, we chose an 11-point scale from German Pain Questionnaire DSF ranging from 0 (no pain) to 10 (pain at its worst) to rate the average pain intensity during the 4 weeks prior [20]. The questionnaire

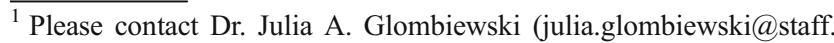
uni-marburg.de) for further information on treatment manual.
} 
was developed by the German Chapter of the International Association for the Study of Pain (www.dgss.org) and has good validity and excellent reliability [20]. Pain intensity was additionally measured over a period of 2 weeks prior to treatment, at posttreatment and follow-up using a pain diary. Subjects rated the pain intensity four times a day on a scale from 0 (no pain) to 10 (pain at its worst). For further analyses, data were averaged to generate a pain index score. The use of analgesics is also recommended as a possible pain-related outcome measure. Patients were asked to write down all drugs taken concurrently during the last 6 months at pretreatment, posttreatment and follow-up. A research assistant, trained by two experienced pain therapists, rated the medicines as "analgesics/adjuvant analgesics" or "others". The outcome measure was the self-reported number of pain drugs taken concurrently over a period of 6 months.

Physical functioning measures Pain disability was measured using the Pain Disability Index (PDI) [21]. The PDI is a measure of interference of chronic pain with functioning in each of seven life areas, generating a maximum global disability score of 7 . The PDI is associated with measures of pain severity, affective distress and activity restriction, and has good psychometric properties [22]. Health-related quality of life was assessed by Health-Related Life Satisfaction Scale (FLZ) [23] (e.g. "I am satisfied with my physical health"). This questionnaire is commonly used in Germany to assess quality of life in medically ill patients [24]. It has good reliability and validity [23].

Emotional functioning measures Depression was measured with the Beck Depression Inventory (BDI) [25], a 21-item self-report questionnaire used to assess the affective, cognitive, motivational and physiological symptoms of depression. The BDI has been shown to have good psychometric properties, also in German samples [26]. The BDI is recommended as core outcome measure of emotional functioning in chronic pain clinical trials (without adjustment for presumed confounding by somatic symptoms) [14]. To assess patients' global retrospective perception of treatment effects at posttreatment and followup, we chose a 5-point transition question ("How are you feeling now (according to your pain problem) compared with when you started treatment?") with the following answer alternatives: "much better", "a little better", "no change", "a little worse", or "much worse". This kind of transition question was found to be a sensitive descriptor of overall effect of pain treatment compared to several measurements, [27]. Patients' satisfaction with treatment was assessed by two 5-point items ("Therapy was helpful (to deal with my pain problem)" and "I would recommend this treatment to a friend") with the following answer alternatives: "yes" "probably", "undecided", "not really" and "no, not at all".

Symptoms and adverse events Potential adverse effects were assessed using the pain intensity questionnaire and the transition question. We defined an increase of pain intensity of 30 or more percent $[14,28]$ and answering the transition question with "little worse" or "much worse" as a change for the worse [27].

Additional measures In addition to the recommendations made by IMMPACT, we also considered coping strategies and health care utilisation as outcome measures. Cognitive and behavioural coping was assessed using the Coping Strategies Scale from the Coping Strategies and PainRelated Distress Questionnaire (FESV) [29] (e.g. cognitive coping item: "when I am in pain, I do not give up", behavioural coping item: "when I am in pain, I do some relaxation training"). A sum score was used. High sum score means good behavioural and cognitive coping strategies. The FESV has been shown to have satisfactory psychometric properties and is commonly used in Germanspeaking countries. Health care use was operationalised by the self-reported number of doctor visits (for pain) in the 6 months prior to treatment, prior to posttreatment and prior to follow-up.

Demographic variables Demographic variables were obtained from a biographic questionnaire as well as from German Pain Questionnaire (DSF) [20] at pretreatment. Comorbidity was assessed by a structured clinical interview based on the DSM-IV during the first session.

\section{Statistical Analyses}

The Statistical Package for the Social Sciences (SPSSTM, Windows version 12) was used. Data were screened for normal distribution. Single missing values were imputed using EM algorithm. In the case where there was more than $50 \%$ missing values for the first measure, the person was excluded from the analysis ( $n=3$; see Fig. 1).

Pain intensity was chosen as primary outcome measure as recommended by IMMPACT [14]. Secondary outcomes were only interpreted in the case of significant primary outcomes.

We used the first wave of study samples (see Fig. 1) to analyse general efficacy of the active treatments compared with the WLC, while we used the complete data sets of treated patients to compare differential effects of the two active treatments. The groups in the "first wave" were independent. For the first analyses, we merged patients that were allocated to CBT-B and CBT in the first treatment 
wave to compare them with patients of the WLC. The purpose of combining these two groups was to avoid testing the hypothesis of group differences between CBT and CBT-B twice.

An intention-to-treat approach using the last value carried forward was used including all randomised patients with available baseline measures even if they dropped out of the study. First, the two groups were compared on descriptive statistics and baseline measures with chi-square tests for dichotomous variables and two sample $t$ tests for continuous variables. Second, we used regression analyses to compute residualised variable change scores (pretreatment vs. posttreatment) for all principal quantitative outcome measures. Third, to test hypothesis A, a 2 (Group) $\times 8$ (Measures) multivariate analysis of variance (MAN-
OVA) was performed to determine treatment effects. Additionally, treatment effect sizes (Cohen's $d$ ) were calculated. The ratio of patients that were clinically significantly improved on the primary outcome measure (pain intensity) was determined for both groups and compared using the chi-square test. Following the recommendations of Farrar et al. [30] and Dworkin et al. [14], a pain patient was designated as significantly improved if she or he obtained a reduction in pain intensity of at least $30 \%$ (so-called "responder"). The ratios of patients that obtained an increase of pain intensity of $30 \%$ and more were also compared between both groups.

To test hypothesis B, we compared all patients that were allocated to CBT-B or CBT in both randomization waves. Patients receiving treatment after the waiting period were
Table 1 Demographic characteristics for the total group and by treatment condition
Data are expressed as numbers (\%) or as $M(\mathrm{SD})$

$C B T-B$ cognitive-behavioural treatment with additional biofeedback elements, $C B T$ cognitive-behavioural treatment, $D S M-I V$ Diagnostic and Statistical Manual of Mental Disorders

\begin{tabular}{|c|c|c|c|}
\hline \multirow[t]{2}{*}{ Characteristics } & \multicolumn{3}{|l|}{ Treatment condition } \\
\hline & Total group $(N=116)$ & CBT-B $(n=62)$ & $\operatorname{CBT}(n=54)$ \\
\hline \multicolumn{4}{|l|}{ Sex } \\
\hline Male & $39(33.6)$ & $21(33.9)$ & $18(33.3)$ \\
\hline Female & $77(66.4)$ & $41(66.1)$ & $36(66.7)$ \\
\hline \multicolumn{4}{|l|}{ Age (years) } \\
\hline Mean & $48.8(11.7)$ & $48.9(10.5)$ & $48.6(13.1)$ \\
\hline \multicolumn{4}{|l|}{ Living situation } \\
\hline Living alone & $22(25.5)$ & $13(21)$ & $9(16.7)$ \\
\hline Cohabiting & $94(74.5)$ & $49(79)$ & $45(83.3)$ \\
\hline \multicolumn{4}{|l|}{ Highest education } \\
\hline Less than high school & $89(84.5)$ & $46(77.4)$ & $50(92.6)$ \\
\hline High school/College & $18(15.5)$ & $14(22.6)$ & $4(7.4)$ \\
\hline \multicolumn{4}{|l|}{ Current employment } \\
\hline Working or in schooling / training & $71(61.2)$ & $42(67.7)$ & $29(53.7)$ \\
\hline \multicolumn{4}{|l|}{ Currently able to work } \\
\hline Yes & $43(60.6)$ & $26(61.9)$ & $17(58.6)$ \\
\hline No & $28(39.4)$ & $16(38.1)$ & $12(41.4)$ \\
\hline Unemployed, retired or homemakers & $26(22.4)$ & $10(16.1)$ & $16(29.6)$ \\
\hline Early retirement due to pain & $19(16.4)$ & $10(16.1)$ & $9(16.7)$ \\
\hline \multicolumn{4}{|l|}{ Pain duration (years) } \\
\hline Mean & $8.1(8.7)$ & $7.7(8.3)$ & $8.6(9.3)$ \\
\hline \multicolumn{4}{|l|}{ At least one surgery due to pain } \\
\hline Yes & $50(43.1)$ & $27(43.5)$ & $23(42.6)$ \\
\hline No & $66(56.9)$ & $35(56.5)$ & $31(57.4)$ \\
\hline \multicolumn{4}{|l|}{ Daily analgesic intake } \\
\hline Yes & $102(87.9)$ & $52(83.9)$ & $50(92.6)$ \\
\hline No & $14(12.1)$ & $10(16.1)$ & $4(7.4)$ \\
\hline \multicolumn{4}{|l|}{ DSM-IV depression diagnosis } \\
\hline Yes & $37(31.9)$ & $18(29)$ & $19(35.2)$ \\
\hline No & $79(68.1)$ & $44(71)$ & $35(64.8)$ \\
\hline \multicolumn{4}{|l|}{ DSM-IV personality disorder diagnosis } \\
\hline Yes & $32(27.6)$ & $18(29)$ & $14(25.9)$ \\
\hline No & $84(72.4)$ & $44(71)$ & $40(74.1)$ \\
\hline
\end{tabular}


merged with patients receiving the corresponding treatment immediately after baseline assessment. For each of the eight continuous variables, a 2 (Group) $\times 3$ (Time) repeated measures analysis of covariance (ANCOVA; Greenhouse-Geisser corrected) was computed with Group (CBT-B vs. CBT) as the between factor, baseline measure as covariate and Time (pretreatment, posttreatment and follow-up) as the repeated measure. Superiority of CBT-B in comparison to CBT would result in significant Group $\times$ Time interactions. Treatment effect sizes and confidence intervals were calculated for both groups for pretreatment versus posttreatment and pretreatment versus follow-up, respectively.

A similar procedure was applied for the transition questions and the two items on patients' satisfaction with treatment. For both groups - for pretreatment versus posttreatment and pretreatment versus follow-up - the ratios of patients who reported feeling "much better" or "a little better" and who answered the satisfaction questions with "yes" or "probably" were compared with chi-square tests. Accordingly, the ratios of patients who reported to feel "a little worse" or "worse" and who answered the satisfaction questions with "not really" or "no, not at all" were also compared.

\section{Results}

Enrolment, Participant Characteristics and Baseline Analyses

Figure 1 illustrates the flow of participants through the study. One hundred sixteen patients were randomised to
CBT-B, CBT and WLC and were considered as the intention-to-treat sample. After waiting time, patients from WLC were randomised to CBT-B and CBT and received the corresponding treatment.

Of the 128 participants who initiated treatment, 23 (18\%) were classified as dropouts. A dropout was defined as any individual who attended at least one session but did not complete the programme (until session 17). CBT-B and CBT did not differ significantly on dropout rates, $\chi^{2}(1, N=$ $116)=.72, p=.4$. An in-depth analysis of dropout reasons and a comparison of dropouts and completers will be reported elsewhere. Sixteen patients $(18.6 \%)$ were lost to follow-up. CBT-B and CBT did not significantly differ on lost to follow-up rates. Adherence to treatment was high, with only ten single cases in which completers did not keep an appointment without timely cancellation.

Demographic information and pain history characteristics of the final randomised sample are presented in Table 1. CBT-B and CBT together compared with WLC (as used for the analysis of general efficacy) did not differ on any descriptive statistics and baseline measures or dropout rates. CBT-B and CBT (as used for the analysis of superiority of CBT-B over CBT) also did not differ on any descriptive statistics and most of the baseline measures except for significantly higher pain disability scores (PDI) in CBT group at pretreatment, $t(114)=-2.27, p<.05$.

There were no differences between the four therapists concerning the effects on outcome variables or dropout rates. There were no differences in method of subject recruitment (i.e. outpatient anaesthesiology centres, general practitioners and self-referral) between CBT-B and CBT against WLC as well as CBT-B against CBT.

Table 2 Group means (and standard deviations) and effect sizes (and confidence intervals) on main outcome measures before and after treatment for the intention-to-treat study groups

\begin{tabular}{|c|c|c|c|c|c|c|}
\hline \multirow[t]{2}{*}{ Measure } & \multicolumn{2}{|c|}{$\mathrm{CBT}+\mathrm{CBT}-\mathrm{B}(n=65)$} & \multicolumn{2}{|c|}{ Waitlist $(n=51)$} & \multirow[b]{2}{*}{ ES } & \multirow[b]{2}{*}{$F$ (group) } \\
\hline & Pretherapy & Posttherapy & Pretherapy & Posttherapy & & \\
\hline Pain Intensity Questionnaire $(0-10)$ & $5.9(1.7)$ & $4.6(1.9)$ & $6.3(1.7)$ & $5.73(1.7)$ & .66 & $F(1,114)=9.11 *$ \\
\hline Pain diary $(0-10)$ & $4.5(1.9)$ & $3.9(1.9)$ & $4.8(2)$ & $4.9(1.8)$ & .55 & $F(1,114)=8.15^{*}$ \\
\hline Number of pain drugs taken concurrently & $4.6(2.4)$ & $3.4(2.2)$ & $3.9(2.7)$ & $3.9(3)$ & .17 & $F(1,114)=7.41 *$ \\
\hline Pain Disability Index & $4.6(2.1)$ & $3.9(2.2)$ & $4.6(1.9)$ & $4.4(2)$ & .25 & $F(1,114)=4.26^{*}$ \\
\hline Health-Related Life Satisfaction Scale & $23.1(8.2)$ & $27.7(8.9)$ & $23.2(7.4)$ & $24.9(8.1)$ & .35 & $F(1,114)=5.47^{*}$ \\
\hline Beck Depression Inventory & $16.3(9.6)$ & $13.3(10.2)$ & $15.7(7.6)$ & $15.1(7.5)$ & .24 & $F(1,114)=5.33^{*}$ \\
\hline Coping Strategies Scale & $69.8(21.9)$ & $87.3(23.6)$ & $71.2(17.1)$ & $72.8(18.6)$ & .80 & $F(1,114)=15.18^{* *}$ \\
\hline Number of doctor visits & $11(9)$ & $7.7(7.5)$ & $8.4(6.1)$ & $8.2(6.4)$ & .1 & $F(1,114)=1.84$ \\
\hline
\end{tabular}

Values are expressed as $M(\mathrm{SD})$. For all scales, except for the Health-Related Life Satisfaction Scale and the Coping Strategies Questionnaire, a lower score is better

$C B T-B$ cognitive-behavioural treatment with additional biofeedback elements, $C B T$ cognitive-behavioural treatment, $E S$ effect size

$* p<.05 * * p<.001$ 
General Efficacy

MANOVA revealed the expected significant main effects for Group for all residualised change scores except for number of doctor visits (see Table 2). The effect sizes in Table 2 refer to the difference between the two treatments versus WCL. Additionally, in the CBT-B plus CBT group, significantly more patients (22 of $65=33.85 \%$ ) than in the WLC group (seven of $51=13.73 \%$ ) were classified as clinically significantly improved, $\chi^{2}(1, N=116)=22.21, p$ $<.001$. In CBT-B plus CBT group, two of 65 patients (3.08\%) obtained an increase of pain intensity of at least $30 \%$ from pretreatment to posttreatment, while in the WLC group, five of 51 patients $(9.8 \%)$ worsened in terms of average pain intensity, $\chi^{2}(1, N=116)=3.32, p=.07$.

\section{Comparison of CBT-B Versus CBT}

\section{Intention-to-Treat Analyses}

Table 3 shows means, standard deviations and effect sizes for continuous outcome measures for pretreatment, posttreatment and follow-up for CBT-B and CBT. Repeated measures ANCOVAS with baseline measure as a covariate showed that there were significant main effects of time on primary outcome measure: Pain Intensity Questionnaire, $F$ $(1.57,177.98)=3.45, p=.043$. There were also significant main effects of time and on some of secondary outcome measures: pain diary, $F(1.85,207.08)=3.3, p=0.041$; Health-Related Life Satisfaction Scale, $F(1.63,184.61)=$ $22.26, p<.001$; number of pain drugs taken concurrently: $F(1.9,133.32)=1.29, p=.28$; Coping Strategies Scale, $F$ $(1.96,221.12)=58.73, p<.001$; number of doctor visits, $F$ $(1.66,186.64)=8.8, p<.001$. There were no further significant effects. There was no significant Group $\times$ Time interaction effects concerning primary outcome measure, revealing that the efficacy of the two treatments was highly comparable: Pain Intensity Questionnaire, $F$ $(1.57,177.98)=.22, p=.75$. Only one secondary outcome effect was significant: Patients in the CBT-B condition visited their doctors less frequently than patients in the CBT condition at 6-month follow-up: number of doctor visits, $F(1.66,186.64)=4.13, p=0.024$. This will not be further interpreted due to non significant effects concerning primary outcome. There were no further significant effects. Table 4 shows comparisons of ratios of clinically significantly improved patients, patients who significantly deteriorated, patients' retrospective perception of treatment effects and treatment satisfaction for pretreatment versus posttreatment and pretreatment versus follow-up for CBT-B and CBT. Chi-square tests revealed no differences concerning these measures between CBT-B and CBT.

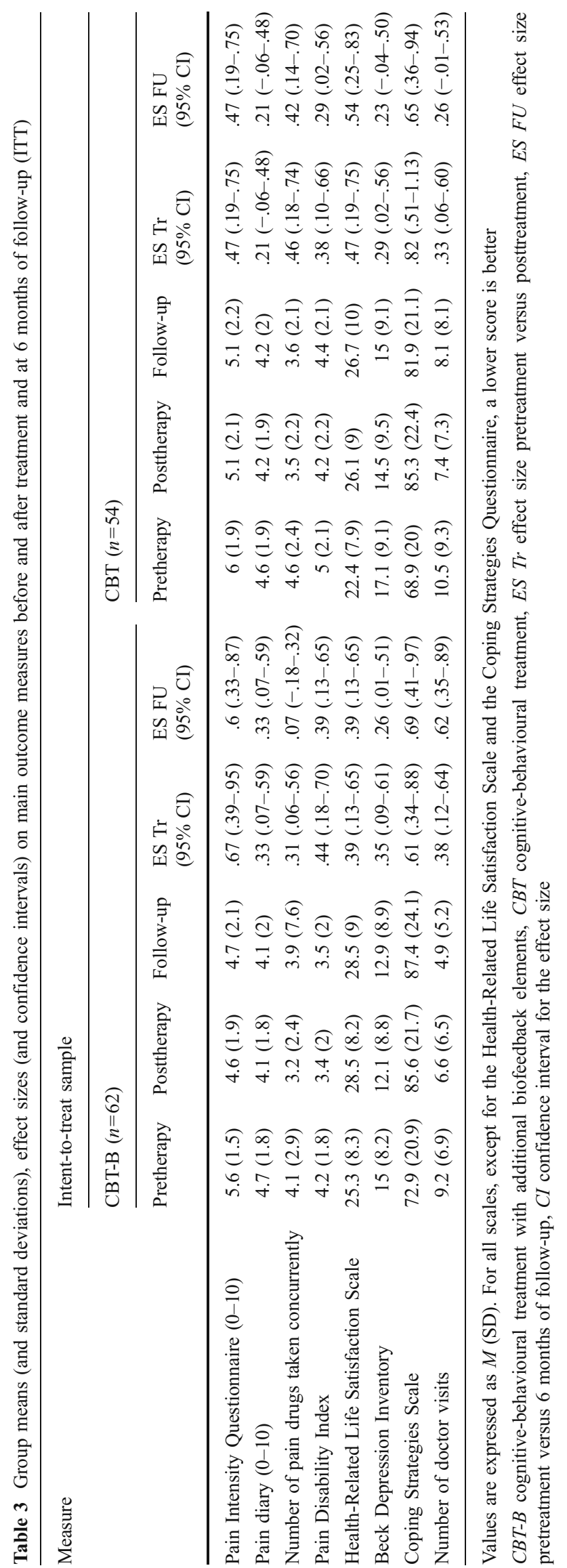


Table 4 Comparison of numbers (and percentages) of clinically significantly improved patients, clinically significantly worsened patients, patients' retrospective perception of treatment effects and treatment satisfaction for pretreatment versus posttreatment and posttreatment versus follow-up for CBT-B and CBT

\begin{tabular}{|c|c|c|c|c|c|c|}
\hline \multirow[t]{2}{*}{ Measure } & \multicolumn{3}{|c|}{ Pretherapy versus posttherapy } & \multicolumn{3}{|c|}{ Prettherapy versus FU } \\
\hline & CBT-B & CBT & $\chi^{2}$ & CBT-B & CBT & $\chi^{2}$ \\
\hline Average pain decrease of at least $30 \%$ (intent-to-treat) & $18(29)$ & $16(29.6)$ & $\chi^{2}(1, N=116)=.01$ & $18(29)$ & $13(24.1)$ & $\chi^{2}(1, N=116)=.64$ \\
\hline Average pain increase of at least $30 \%$ (intent-to-treat) & $2(3.2)$ & $4(7.4)$ & $\chi^{2}(1, N=116)=1.58$ & $3(4.8)$ & $3(5.6)$ & $\chi^{2}(1, N=116)=.07$ \\
\hline Average pain decrease of at least $30 \%$ (completer) & $18(36.7)$ & $16(38.1)$ & $\chi^{2}(1, N=91)=.04$ & $18(36.7)$ & $13(31)$ & $\chi^{2}(1, N=91)=.58$ \\
\hline Average pain increase of at least $30 \%$ (completer) & $2(4.1)$ & $4(9.5)$ & $\chi^{2}(1, N=91)=3.14$ & $3(6.1)$ & $3(7.1)$ & $\chi^{2}(1, N=91)=.08$ \\
\hline $\begin{array}{l}\text { Rating of global improvement: feeling "a little better" or } \\
\text { "much better" }\end{array}$ & $36(73.5)$ & $32(76.2)$ & $\chi^{2}(1, N=91)=.16$ & $29(70.7)$ & $24(75)$ & $\chi^{2}(1, N=73)=.4$ \\
\hline $\begin{array}{l}\text { Rating of global improvement: feeling "a little worse" or } \\
\text { "worse" }\end{array}$ & $2(4.1)$ & $3(7.1)$ & $\chi^{2}(1, N=91)=.68$ & $1(2.4)$ & $3(9.4)$ & $\chi^{2}(1, N=73)=.2 .3$ \\
\hline Therapy was helpful: "yes" or "probably" & $46(93.9)$ & $38(95)$ & $\chi^{2}(1, N=91)=.13$ & $40(97.6)$ & $28(96.6)$ & $\chi^{2}(1, N=73)=.12$ \\
\hline $\begin{array}{l}\text { I would recommend treatment to a friend: "yes" or } \\
\text { "probably" }\end{array}$ & $46(93.9)$ & $37(92.5)$ & $\chi^{2}(1, N=91)=.13$ & $40(97.6)$ & $28(96.6)$ & $\chi^{2}(1, N=73)=.12$ \\
\hline
\end{tabular}

Values are expressed as absolute numbers $(\%)$. For ratings of global improvement and treatment satisfaction, only completer data were included $C B T-B$ cognitive-behavioural treatment with additional biofeedback elements, $C B T$ cognitive-behavioural treatment, $F U 6$ months of follow-up

\section{Discussion}

The present study was designed to examine the impact of biofeedback tools within cognitive-behavioural treatment of chronic back pain.

The hypothesis that CBT and CBT-B for chronic pain would be effective in comparison to WLC received support across most measures of pain intensity and related symptomatology. But, contrary to our expectations, including biofeedback to cognitive-behavioural treatment did not result in improved outcomes at either posttreatment or at 6 months of follow-up, which confirms earlier reports of Newton-John et al. [7] including a small sample of mildly disabled patients. We could confirm this finding for a larger and highly disabled sample, comparable to sample described by McCracken et al. [31].

Our results indicate that EMG biofeedback might be not necessary to reach improved efficacy of CBT in chronic back pain. However, some caution should be exercised when interpreting the results of the present study. Flor and Birbaumer [5] demonstrated that differences between EMG biofeedback and CBT tend to increase over time, with the most marked differences appearing at 2-year follow-up. These effects of biofeedback probably occur due to continued practice and application of self-regulation skills at home causing long-term changes of habits concerning postures or tensed muscles. Therefore, the possibility remains that differences will be found at 2-year follow-up. Further, the lack of significant difference between treatments may have been due to unknown non-specific factors or ceiling effects that occur when two powerful active treatments are compared. The sample size could also be insufficient to detect some smaller differences between groups: for some variables in our study, mean scores of change differed between the two interventions, despite not reaching statistical significance. Furthermore, it should be kept in mind that if two treatments have comparable efficacy, this does not imply that the same patients would benefit from both treatments, and that the process of change is identical. Further predictor and process analyses are needed to define criteria outlining who benefits from CBT and who benefits from additional biofeedback components. According to the verbal feedback of patients of the biofeedback group, it seems that they were more motivated to accept psychological interventions, despite an initial reluctance. However, we were unable to show any specific benefits in acceptance or outcome.

The present study has several limitations. We did not rate video tapes of therapy sessions systematically, although all treatments were supervised and examples of videotapes were checked for treatment fidelity in supervision sessions. The allocation of patients to therapists was not fully randomised. All included measures were self-reported and thus could be biased by social desirability. Neither patients nor therapists were blinded to the purpose of the study, although this apparently did not influence outcomes in favour of the main hypothesis. These and other shortcomings could have had an impact on findings and should be considered while interpreting the data.

Our study also exhibits several strengths. We could meet and report on most of the requirements for efficacy studies [15] by concurrently guarantying high generalisability of the results as demanded for effectiveness studies. We provided convincing external validity, as our participants 
were typical referrals from GPs and medical specialists treated through typical treatment paths of the health care system. Many patients with low education status, comorbid depression, or high sickness leave, as well as half of the patients with histories of back surgery are just a few features underlining the external validity of our results. Additionally to comparing two treatments, we added a waitlist control group to enhance the quantitative evaluation of treatment efficacy. To our knowledge, our study is the first one designed to answer the important question of additional effects of biofeedback in chronic pain with a sufficiently powered sample size.

When interpreting the effects of cognitive-behavioural treatments, we should keep in mind that chronic pain is caused and maintained by a complex interaction of many individual, psychosocial and occupational factors [32, 33]. Only parts of these factors can be addressed by psychological interventions. For this reason, the recommended treatment for patients with chronic back pain is multidisciplinary intervention incorporating multiple treatment components, such as physical exercises and biopsychosocial and cognitive-behavioural interventions [34].

In conclusion, biofeedback ingredients did not lead to improved outcome of a psychological intervention. Both treatment packages are possible and powerful options to improve the health status of chronic back pain patients.

Conflicts of interest There are no conflicts of interest.

\section{References}

1. Schmidt CO, Raspe H, Pfingsten M, Hasenbring M, Basler HD, Eich W, et al. Back pain in the German adult population: prevalence, severity, and sociodemographic correlates in a multiregional survey. Spine. 2007;32:2005-11.

2. Hoffman BM, Papas RK, Chatkoff DK, Kerns RD. Meta-analysis of psychological interventions for chronic low back pain. Health Psychol. 2007;26:1-9.

3. van Tulder MW, Ostelo R, Vlaeyen JW, Linton SJ, Morley SJ, Assendelft WJ. Behavioral treatment for chronic low back pain: a systematic review within the framework of the Cochrane Back Review Group. Spine. 2001;26:270-81.

4. Nigl AJ. A comparison of binary and analog EMG feedback techniques in the treatment of low back pain. Am J Clin Biofeedback. 1981;4:25-31.

5. Flor H, Birbaumer N. Comparison of the efficacy of electromyographic biofeedback, cognitive-behavioral therapy, and conservative medical interventions in the treatment of chronic musculoskeletal pain. J Consult Clin Psychol. 1993;61:653-8.

6. Kroener-Herwig B, Beck A. An exploratory study of biofeedback for chronic low back pain. Br J Th Rehab. 2000;7:134-42.

7. Newton-John TR, Spence SH, Schotte D. Cognitive-behavioural therapy versus EMG biofeedback in the treatment of chronic low back pain. Behav Res Ther. 1995;33:691-7.
8. Hemingway MA, Biedermann HJ, Inglis J. Electromyographic recordings of paraspinal muscles: variations related to subcutaneous tissue thickness. Biofeedback Self Regul. 1995;20:39-49.

9. Keefe FJ, Shapira B. EMG-assisted relaxation training in the management of chronic low back pain. Am J Clin Biofeedback. 1981;4:93-103.

10. Nouwen A. EMG biofeedback used to reduce standing levels of paraspinal muscle tension in chronic low back pain. Pain. 1983;17:353-60.

11. Stuckey SJ, Jacobs A, Goldfarb J. EMG biofeedback training, relaxation training, and placebo for the relief of chronic back pain. Percept Mot Skills. 1986;63:1023-36.

12. Bush C, Ditto B, Feuerstein M. A controlled evaluation of paraspinal EMG biofeedback in the treatment of chronic low back pain. Health Psychol. 1985;4:307-21.

13. Large RG, Lamb AM. Electromyographic (EMG) feedback in chronic musculoskeletal pain: a controlled trial. Pain. 1983;17:167-77.

14. Dworkin RH, Turk DC, Farrar JT, Haythornthwaite JA, Jensen MP, Katz NP, et al. Core outcome measures for chronic pain clinical trials: IMMPACT recommendations. Pain. 2005;113:9-19.

15. Yates SL, Morley S, Eccleston C, Williams ACD. A scale for rating the quality of psychological trials for pain. Pain. 2005;117:314-25.

16. Cohen J. Statistical power analysis for the behavioral sciences. 2nd ed. Hillsdale: Lawrence Erlbaum Associates; 1988.

17. Faul F, Erdfelder E, Lang AG, Buchner A. G*Power 3: a flexible statistical power analysis program for the social, behavioral, and biomedical sciences. Behav Res Methods. 2007;39:175-91.

18. Flor H, Turk DC. Psychophysiology of chronic pain: do chronic pain patients exhibit symptom-specific psychophysiological responses? Psychol Bullet. 1989;105:215-59.

19. Cram JR, Kasman GS, Holtz J. Introduction to surface electromyography. Gaithersburg: Aspen; 1998.

20. Nagel B, Gerbershagen HU, Lindena G, Pfingsten $M$. Entwicklung und empirische Uberprufung des Deutschen Schmerzfragebogens der DGSS. Schmerz. 2002;16:263-70.

21. Pollard CA. Preliminary validity study of the Pain Disability Index. Percept Mot Skills. 1986;59:974.

22. Tait RC, Chibnall JT, Krause S. The pain disability index: psychometric properties. Pain. 1990;40:171-82.

23. Myrtek M. Life satisfaction, illness behaviour, and rehabilitation outcome: results of a one year follow-up study with cardiac patients. Int J Rehabil Res. 1987;10:373-82.

24. Myrtek M, Fichtler A, Konig K, Brugner G, Muller W. Differences between patients with asymptomatic and symptomatic myocardial infarction: the relevance of psychological factors. Eur Heart J. 1994;15:311-7.

25. Beck AT, Steer RA, Garbin MG. Psychometric properties of the Beck Depression Inventory: twenty-five years of evaluation. Clin Psych Rev. 1988;8:77-100.

26. Hautzinger M. Das Beck-Depressioninventar (BDI) in der Klinik The German version of the Beck Depression Inventory (BDI) in clinical use. Nervenarzt. 1991;62:689-96.

27. Lauridsen HH, Hartvigsen J, Korsholm L, Grunnet Nilsson N, Manniche C. Choice of external criteria in back pain research: Does it matter? Recommendations based on analysis of responsiveness. Pain. 2007;131:112-20.

28. Morley S, Williams A, Hussain S. Estimating the clinical effectiveness of cognitive behavioural therapy in the clinic: Evaluation of a CBT informed pain management programme. Pain. 2008;137:670-80.

29. Geissner E. Dimensionen der Verarbeitung chronischer Schmerzen-Eine Replikationsstudie. Z Klin Psychol Psychopathol Psychother. 1992;40:20-33. 
30. Farrar JT, Young JP Jr, LaMoreaux L, Werth JL, Poole RM. Clinical importance of changes in chronic pain intensity measured on an 11-point numerical pain rating scale. Pain. 2001;94:149-58.

31. McCracken LM, Vowles KE, Eccleston C. Acceptance-based treatment for persons with complex, long standing chronic pain: a preliminary analysis of treatment outcome in comparison to a waiting phase. Behav Res Ther. 2005;43:1335-46.
32. van Tulder M, Koes B, Bombardier C. Low back pain. Clin Rheumatol. 2002;16:761-75.

33. Linton SJ. A review of psychological risk factors in back and neck pain. Spine. 2000;25:1148-56.

34. Buchner M, Zahlten Hinguranage A, Schiltenwolf M, Neubauer E. Therapy outcome after multidisciplinary treatment for chronic neck and chronic low back pain: a prospective clinical study in 365 patients. Scand J Rheumatol. 2006;35:363-7. 
Copyright of International Journal of Behavioral Medicine is the property of Springer Science \& Business Media B.V. and its content may not be copied or emailed to multiple sites or posted to a listserv without the copyright holder's express written permission. However, users may print, download, or email articles for individual use. 